\begin{tabular}{l} 
SCIENCE \& TECHNOLOGY \\
Journal homepage: http://www.pertanika.upm.edu.my/ \\
\hline PERTANIKA
\end{tabular}

\title{
A New Parametric Function-Based Dynamic Lane Changing Trajectory Planning and Simulation Model
}

\author{
Md. Mijanoor Rahman ${ }^{1,2}$, Mohd. Tahir Ismail ${ }^{1}$, Norhashidah Awang ${ }^{1}$ and \\ Majid Khan Majahar Ali ${ }^{1 *}$ \\ ${ }^{1}$ School of Mathematical Sciences, Universiti Sains Malaysia, 11800 USM, Pulau Penang, Malaysia \\ ${ }^{2}$ Department of Mathematics, Mawlana Bhashani Science and Technology University, Tangail-1902, Bangladesh
}

\begin{abstract}
Lane-changing (LC) problem may cause serious accidents or create a painful traffic jam at multi-lane roads. Existing LC simulation model was created with some limitations (less fitted, without velocity and acceleration profiles, high curvature) by using well known trajectory curve such as Hyperbolic Tangent Curve (HTC), Sine-Based Curve (SC), Polynomial Curve (PC). In this study, a new parametric curve had been proposed by using curvilinear coordinate system and fitted against Next Generation Simulation (NGSIM) real dataset. Further, new profiles of velocity and acceleration were designed using the proposed LC trajectory curve. The curvature of proposed model was zero-based curvature both at LC starting and ending points. This proposed curvature was compared with two models such as HTC and SC. The average root-mean-square-error of proposed model decreased with $1.84 \%$ for left LC and $15.48 \%$ for right LC compared to HTC model and $1.74 \%$ for left LC and $15.60 \%$ for right LC compared to SC model. Similarly, the proposed model for velocity and acceleration profiles improved significantly from PC model. The proposed parametric curve solves the gap and collision points of LC vehicle with a front vehicle and rear vehicle at target lane and can be used in real LC path planning.
\end{abstract}

ARTICLE INFO

Article history:

Received: 20 June 2020

Accepted: 20 October 2020

Published: 22 January 2021

DOI: https://doi.org/10.47836/pjst.29.1.12

$\overline{\text { E-mail addresses: }}$

mijanoor.math@student.usm.my (Md. Mijanoor Rahman)

m.tahir@usm.my (Mohd. Tahir Ismail)

shidah@usm.my (Norhashidah Awang)

majidkhanmajaharali@usm.my (Majid Khan Majahar Ali)

$*$ Corresponding author
Keywords: Acceleration profiles, parametric curve, speed, trajectory planning

\section{INTRODUCTION}

Lane Changing Trajectory (LCT) planning is an important model for identifying and ensuring the safeness in any traffic systems where this model helps to predict the gap acceptance, and to plan LC dynamical 
trajectory about the longitudinal and lateral movements (Yang et al., 2018). The trajectory planning has been developed from more than two decades. Few simulation models were developed for LCT system such as Quintic Bezier Curves (QBC), Spline-Based Curve (SBC), non-smooth Dijkstra algorithm and Multi Order Polynomial Curve (MOPC) for urban and freeway roads. Testing the simulation model by real trajectory data is required for this development, because the model based on simulation data poorly fits in the real traffic data (Zhou et al., 2017).

The QBC is used in LCT planning for shortest-distance and smoothness path, timeoptimal and comfortable journey. Shen et al. (2017) addressed the trajectory planning based on the fifth order QBC for a comfortable journey. They implemented this curve with a few mini-mature vehicles in LC scenarios to test comfort measurement. However, their results were unrealistic as there was no error testing between the proposed path planning and real trajectory planning for longitudinal and lateral path positions. Meanwhile, González et al. (2016) and Kawabata et al. (2013) found that although QBC was very smooth, it was only applied on unicycle trajectory and agreed that high-degree QBC lost flexibility at the trajectory.

The parameters of MOPC are described by acceleration, speed and position constraints. Sometimes, inexperienced driving causes uncomfortable journey at the time of LC. Wang and Zheng (2013) provided a simulation model for LCT planning using MOPC without testing the with real vehicle trajectory. A few researches only assumed that the acceleration and velocity at the starting and ending points were zeros to generate the PC-based lateral trajectory model (Resende \& Nashashibi, 2010; Wang \& Zheng, 2013; You et al., 2015; Ntousakis et al., 2016; Chebly et al., 2017).

Heil et al. (2016) developed the PC-based LCT planning and found the computational cost by using maximum acceleration and overshooting behaviour. Connors and Elkaim (2007) had successfully overcome collision points during LC. They used the trajectory planning based on SBC to overcome any obstacle point. SBC is very important for smoothness at the corner between two straight lines with limitation that the continuous velocity was not possible at this corner point (Sanchez-Reyes \& Chacón, 2018). Therefore, the most of LCT model developed using the MOPC model, but most of the research still only assumed its velocity and acceleration profiles at the starting and ending points were zeros, in which these assumptions were unrealistic. Wang et al. (2018) explored another lateral trajectory path based on SC and a longitudinal trajectory line. Their trajectory model still assumed zeros for lateral velocity and acceleration.

Zhou et al. (2017), modified a reference angle-based trajectory planning model using HTC. The curvature and fitted values were compared with PC and SBC, in which modified HTC performed better than other two models. They suggested that the reference angle could only be determined by vehicle traveling data recorders. So, data extracted from still 
video camera cannot be employed in reference angle adopted trajectory model whereas this type of data is very popular (Wan et al. 2020). Yang et al. (2018) modified the MPOC model in order to determine LCT curve and found that the starting and ending points were non- zeros as assumed previously by other researchers. However, they assumed the vehicle at ending point was parallel to the target lane. If a vehicle is parallel to the target lane, then the lateral velocity should be zero as non-lateral movements. Therefore, that assumption is also unrealistic.

Katrakazas et al., (2015) discovered that there were two important types of findings for LCT problems

1. The best geometric trajectory was necessary for LC vehicle. It indicates that the curvature at every point on the curve was to be as small as possible, besides the curvatures at starting and ending points were nearly zero for comfortable journey.

2. The realistic vehicle dynamical system was very important for path planning. The vehicle LC time versus position, vehicle LC time versus velocity and vehicle LC time versus acceleration should be validated by real trajectory.

In this study, a new parametric trajectory curve, velocity and acceleration profiles were proposed by using curvilinear motion planning. The proposed trajectory curve was fitted with NGSIM data. Further, the curvature of the trajectory curve was calculated and compared with HTC and SC models. Furthermore, the velocity and acceleration profiles were compared with PC model.

\section{METHODS}

\section{The Curvilinear Motion Planning (Rectangular Coordinates)}

Let $X, Y$ be the coordinate frame where the horizontal direction is represented by the $x$-axis and the vertical direction is represented by the $y$-axis. The rectangular coordinate system represents the vehicle's longitudinal and lateral positions, respectively, $X(t)$ and $Y(t)$. So, the position of the vehicle, at any given time $t$, is $P(t)=[X(t), Y(t)]$, where is the position of the cartesian system and $t$ is the time during LC.

\section{Trajectory Curve}

The parametric function for LCT planning is identified due to the nature of the function, which is continuous, low curvature; and because they ensure easy computation and timesaving trajectories. The parameters of this functions represent the positioned coordinates of the LCT with respect to time. But for the aggressive driver, parameters' values must be changed quickly to reach the target point. Further, we propose a lateral velocity and acceleration profiles for vehicle motion. Equation 1 and 2 represent for longitudinal movement and lateral movement respectively to identify velocity and acceleration: 
Longitudinal position (Y. Y. Wang, Pan, Liu \& Feng, 2018),

$$
X(t)=u_{0} t+L_{0} \text {, for } T_{\text {origin }} \leq \mathrm{t} \leq T_{\text {target }}
$$

Lateral position of proposed curve,

$$
Y(t)=\frac{L_{Y D}}{2}\left(\tanh t_{1}-t\right)+L_{Y I D}, \text { for } T_{\text {origin }} \leq \mathrm{t} \leq T_{\text {target }}
$$

where, $u_{o}$ is initial velocity, $L_{o}$ is initial longitudinal position during LC, $T_{\text {origin }}$ and $T_{\text {target }}$ are starting time and ending time of LC respectively. In Equation 2, the parameter $L_{Y D}$ represents to the total lateral displacement during $L C$ wherein it is positive for Left LC (LLC) and negative for Right LC (RLC). The range of total lateral displacement, $L_{Y I D}$ is $[2.8,4]$ for LLC and $[-4,-2.8]$ for RLC scenarios. $L_{Y I D}$ is ordinate of middle position of LC starting and ending points and $t_{1}$ is the such time that vehicle arrives at middle position of starting and ending positions. Where, $\sigma$ is a weighted parameter represents to be estimated. Figure 1 depicts the parameters movement on RLC and LLC scenario using Equations 1 and 2. These parametric functions are fitted the LLC and RLC in order to find LCT in lane width, middle position of LC starting and ending points are initially used, when a driver needs to change the lane. Figures 2 and 3 are time versus RLC and LLC positions respectively using Equation 2 where the vehicle lateral position is decreasing for RLC, and increasing for LLC.

\section{Velocity Profile}

The longitudinal and the lateral positions are represented by Equation 1 and 2 with respect to time respectively. The lateral displacement per unit time was found by taking the derivative with respect to time, then the lateral velocity of the vehicle comes out. Similarly, longitudinal velocity was calculated by differentiation with respect to time.

The vehicle velocity vector is parallel to the tangent line on the position vector of the parametric path, where the lateral velocity is zero and the longitudinal velocity is linear motion before LC. When LC starts, the value of lateral velocity increases, but the longitudinal speed remains in the previous speed. However, for the motion control and path planning of the vehicle, this velocity profile is suitable to overcome the smoothness and curvature limitation.

If a LC starts from zero second and finishes at eighth second, then time interval is $[0,8]$ seconds, but some aggressive vehicles are very fast, and their LC time interval is $[0,6]$ seconds (Zhou et al., 2017). The LC processing starts from time, $t=0$, and the velocity function is increasing until vehicles arrive at the middle position of two lanes. After that, velocity decreases for merging with the target lane. Since, the lateral velocity is not very 


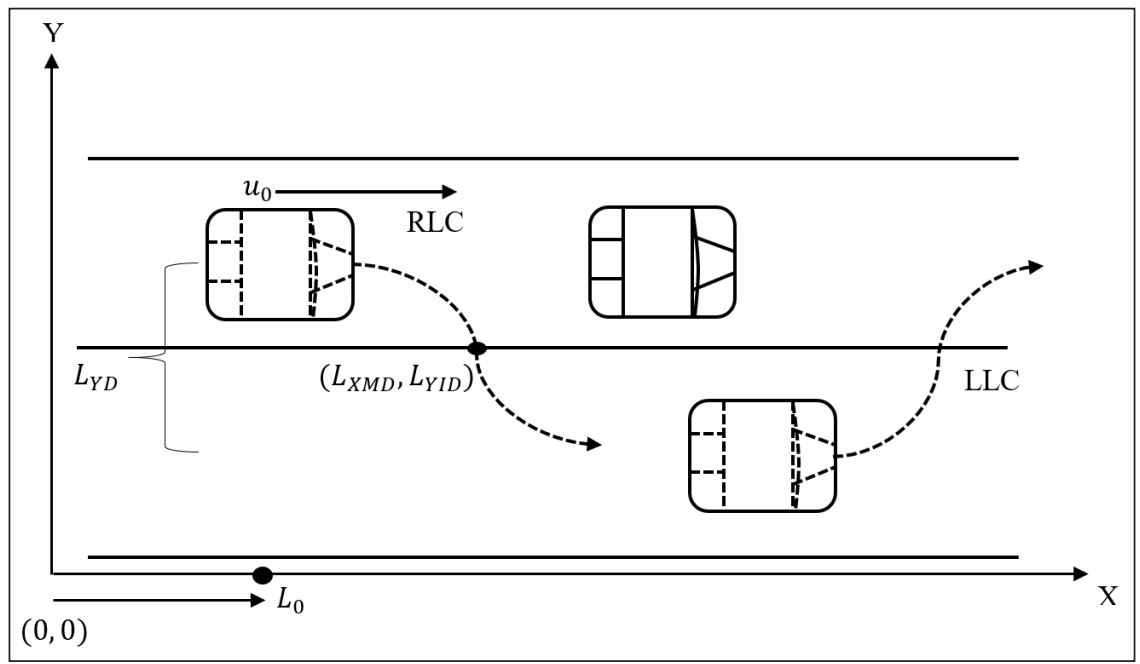

Figure 1. RLC and LLC traffic scenarios

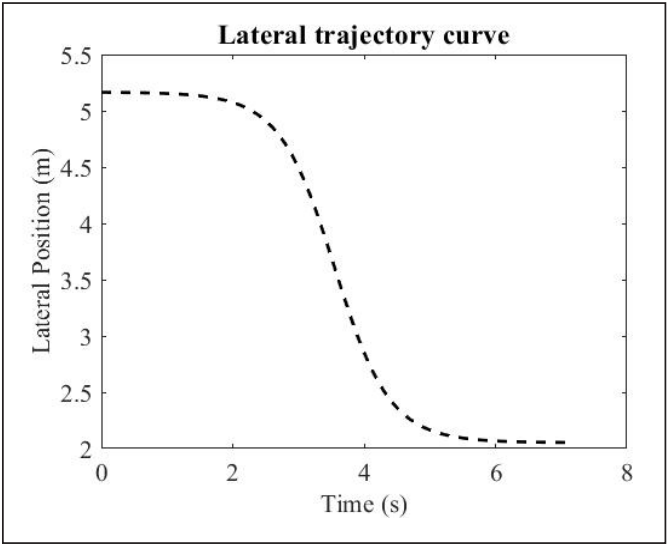

Figure 2. Parametric RLC path planning for lateral position

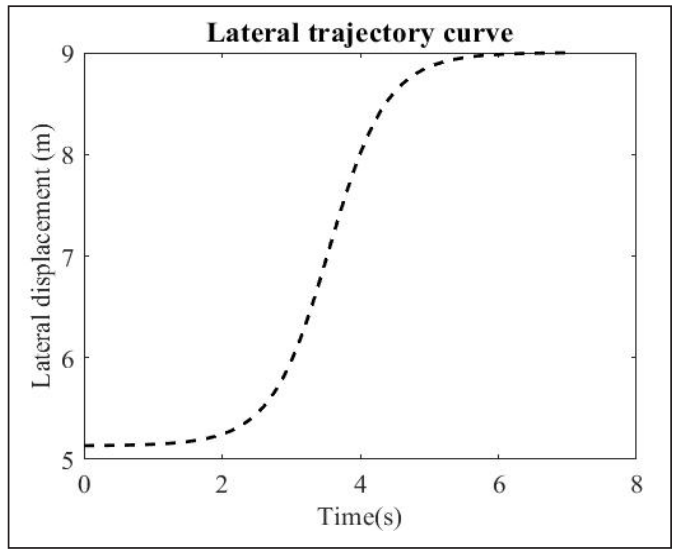

Figure 3. Parametric LLC path planning for lateral position

fast, the total LC velocity mostly depends on the longitudinal velocity for arriving at target lane in time. The longitudinal and lateral velocities shown in Equation 3 and 4 respectively where the Equation 1 and 2 are differentiated with respect to $t$.

Longitudinal velocity,

$$
v_{X}(t)=u_{0} \text {, for } T_{\text {origin }} \leq \mathrm{t} \leq T_{\text {target }}
$$

Lateral velocity,

$$
v_{Y}(t)=-\sigma \frac{L_{Y D}}{2} \operatorname{sech}^{2} \sigma\left(t_{1}-t\right) \text { for } T_{\text {origin }} \leq \mathrm{t} \leq T_{\text {target }}
$$


Figure 4 represents the longitudinal and lateral velocities by using Equations 3 and 4 where longitudinal velocity is parallel to the horizontal line, and lateral velocity is a smooth curve during LC.

\section{Acceleration Profile}

A comfortable journey should adopt low lateral acceleration according to Katrakazas et al. (2015) work. Since, this study uses the smooth lateral velocity and constant longitudinal velocity based on Equation 3 and 4, respectively. The longitudinal and lateral accelerations presented in Equations 5 and 6 respectively by using the differentiation of Equations 3 and 4 during LC.

Longitudinal acceleration,

$$
a_{X}(t)=0 \text { for } T_{\text {origin }} \leq \mathrm{t} \leq T_{\text {target }}
$$

Lateral acceleration,

$$
a_{Y}(t)=\sigma^{2} L_{Y D} \operatorname{sech}^{2} \sigma\left(t_{1}-t\right) \tanh \sigma\left(t_{1}-t\right) \text { for } \quad T_{\text {origin }} \leq \mathrm{t} \leq T_{\text {target }}
$$

Figure 5 represents the longitudinal and lateral acceleration where longitudinal acceleration is zero according to Equation 5, and lateral acceleration is a smooth curve using Equation 6.

\section{DATA PROCESSING}

Dong et al. (2017) had estimated the starting and ending points of LC with less standard deviation and limited mean error from US 101, NGSIM dataset. This data set included the longitudinal and lateral position of LC vehicle with other surrounding vehicles, such as a

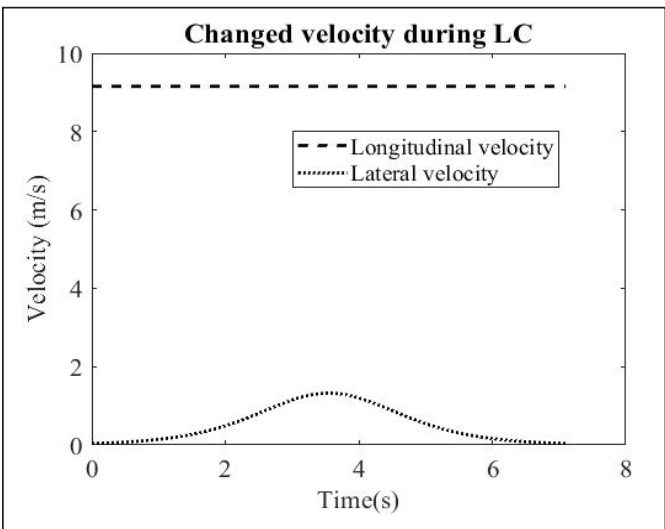

Figure 4. Longitudinal and lateral velocities of the parametric curve LC times

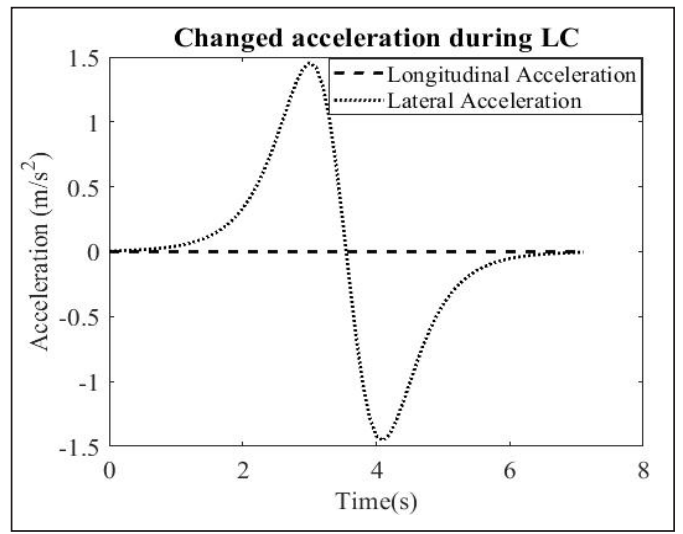

Figure 5. Longitudinal and lateral accelerations of parametric curve during $\mathrm{LC}$ 
front vehicle, lag vehicle on the current lane and front vehicle, rear vehicle on the target lane. This dataset included groups of LC vehicle with surrounding. Every group had five vehicles, such as a front vehicle, lag vehicle on the current lane and front vehicle, rear vehicle on the target lane. The study used 200 LC vehicles to fit the proposed LCT model during LC.

Every vehicle in a group had $20 \mathrm{~s}$ longitudinal and lateral velocity and longitudinal and lateral acceleration profiles time series microscopic data where the data resolution was 0.1 second (Li et al., 2016). This dataset also included the LC starting and ending position of $\mathrm{LC}$ vehicle, and the movement position of other surrounding vehicles. The LC duration depended on the starting and ending points of the trajectory data. Before starting point, the longitudinal and lateral positions were before LC data, and after the ending point, the positioning data were after LC data. During LC, for getting the average longitudinal velocity and lateral velocity of every vehicle, the total longitudinal and lateral displacement was divided by the total time. Similarly, longitudinal acceleration and lateral acceleration were found from longitudinal velocity and lateral velocity respectively.

\section{RESULTS AND DISCUSSION}

As mentioned previously, one of the limitations of reference angle, the value only can be collected from vehicle traveling data recorders, but not from still video camera as in NGSIM. So, Zhou et al. (2017) adopted parameters (average velocity, longitudinal distance and reference angle) were modified using coordinate geometrical system, as shown in Appendix A. In addition, another SC model is proposed by Wang et al. (2018) also shown in Appendix B. Both of HTC and SC were used to compare the efficiency of the proposed parametric curve.

Equations 1 and 2 present a longitudinal and lateral positions of the LC. By using parameters' values- $L_{Y D}$ and $L_{Y I D}, t_{1}, u_{0}$ and $L_{0}$, the proposed curve was fitted to $200 \mathrm{LC}$ vehicles' trajectories. The only parameter's value, $\sigma$ was tested by using initial value 0 to 1 because it was a weighted parameter. So, this study found the more fitted value of $\sigma$ is 0.56. In addition, the fitted curves and real trajectory curves were compared.

\section{Curvature Estimation}

Equation 7 (Léger, 1999) was applied for determining the curvature value of proposed parametric curve, and compared to HTC and SC curves.

$$
\text { Curvature, } k=\frac{y^{\prime \prime}}{\left(1+y^{\prime 2}\right)^{3 / 2}}
$$

where, $y^{\prime}$ and $y^{\prime \prime}$ are first and second differentiations respectively of lateral displacement, $y^{\prime}$ with respect to longitudinal displacement, $x$ by using Equations 1 and 2. The maximum 
values of the curvature were not more than 0.006 for LLC and 0.009 for RLC shown in Figure 6 and Figure 7. These curvature values were nearly zeros at the starting and ending points of the curve. Therefore, the proposed curve provided the comfortable journey according to the small curvatures at the starting and ending points during $\mathrm{LC}$ as a challenge of Katrakazas et al. (2015) study.

The curvature of the proposed curve is shown in Figures 6 and Figure 7 by using Equation 7, and these curvatures are lower than the HTC and SC proposed curvatures. However, by using the LCT in the parametric curve, the driver not only determines the vehicle path, but also follows the longitudinal and lateral velocity and acceleration profiles with respect to their desired dynamics and gaps at the target lane. Many other information can also be collected by using the prediction curve besides the proposed model can generate different trajectories which follow the aggressive and non-aggressive drivers by changing the values of the initial parameters of the prediction curve.

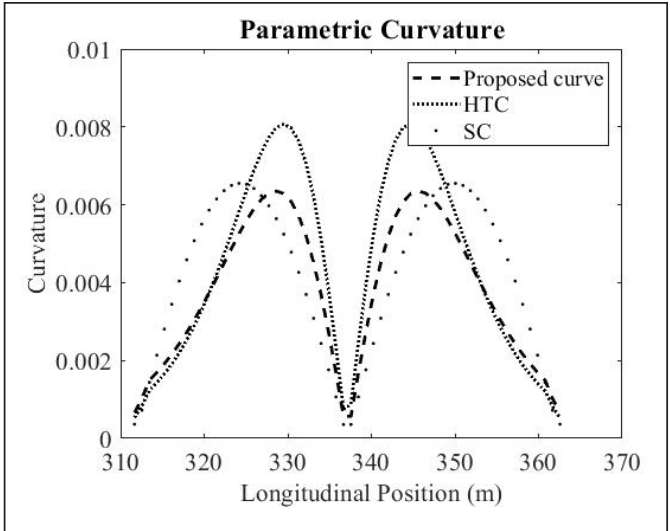

Figure 6. The curvature for LLC

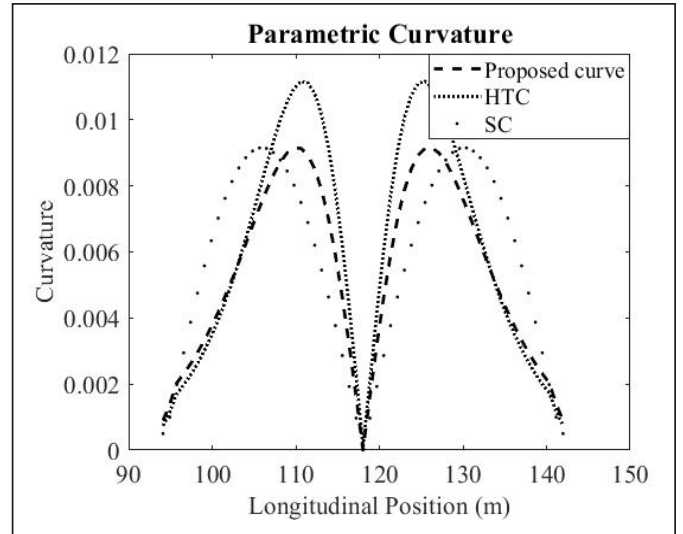

Figure 7. The curvature for RLC

\section{Trajectory Curve Validation}

For validation test, the total of 200 LC real trajectory data (100 LLC and 100 RLC data) were randomly taken from 200 groups of vehicles LC scenarios for NGSIM data. The parameters' values of prediction trajectory curve were used to adjust with the real trajectory vehicle position. Table 1 shows that the average Root Mean Square Error (RMSE) values where the intervals of total lateral distance of RLC and LLC were [3.3, 4.4] meters and [2.7, 5.4] meters respectively. The middle longitudinal point and the middle lateral point were situated in the middle coordinate of the trajectory curve. The middle coordinate changed for every trajectory curve, because the LC position of every trajectory was different. But the ranges of total longitudinal LC distance were $[25,85]$ meters for RLC and $[30,93]$ meters for LLC real data. The total LC time depended on aggressive driving or regular driving. Here, the ranges of total LC time are $[5.5,8.9]$ seconds for RLC and $[4.8,8.8]$ 
seconds for LLC appeared in the dataset. These dynamical parameters of the curve were collected from real dataset to fit this curve with real trajectory planning.

The total 100 LLC and 100 RLC vehicles data were tested by using proposed trajectory model, HTC model and SC model. The HTC and SC models used the Equation 8 and 9 respectively where Equation 8 was adopted in Appendix A, and Equation 9 was adopted in Appendix B. The average RMSE values of used models are presented in Table 1 for comparison of the proposed model. The average RMSE of proposed parametric curve for LLC and RLC were 0.2795 and 0.2179 respectively, where proposed model improved $1.84 \%$ from HTC, and 1.74\% from SC for LLC, and 15.48\% from HTC, and $15.60 \%$ from SC.

Figure 8 shows the lateral displacements of RLC vehicles along our proposed curve, HTC, SC and real trajectories of NGSIM data sets. The real trajectory of a vehicle (randomly selected from 100 groups of vehicles) for RLC are represented. Figure 9 shows the longitudinal movements according to Equation 1 and the same vehicle longitudinal movements. This study used the Wang et al. (2018) proposed longitudinal movements line due to use the determination of the curvature, velocity and acceleration. Figure 8 clearly shows that the lateral displacements of parametric curve are similar to that of the real trajectory curve, and better than the HTC and SC due to movement of the proposed curve and real trajectory.

Table 1

The parametric curve fitting RMSE value using 200 vehicles data

\begin{tabular}{ccccccc}
\hline & $\begin{array}{c}\text { Proposed } \\
(\text { LLC) }\end{array}$ & HTC (LLC) & SC (LLC) & $\begin{array}{c}\text { Proposed } \\
(\text { RLC) }\end{array}$ & HTC (RLC) & SC (RLC) \\
\hline Average RMSE (m) & 0.2795 & 0.2848 & 0.2845 & 0.1719 & 0.2033 & 0.2036 \\
$\begin{array}{c}\text { Improved proposed } \\
\text { model }\end{array}$ & & From & From & & From & From \\
\hline
\end{tabular}

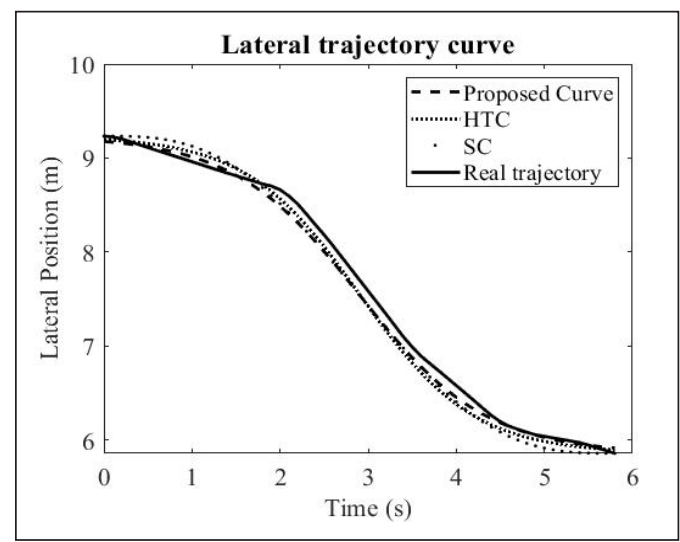

Figure 8. Lateral movement of RLC

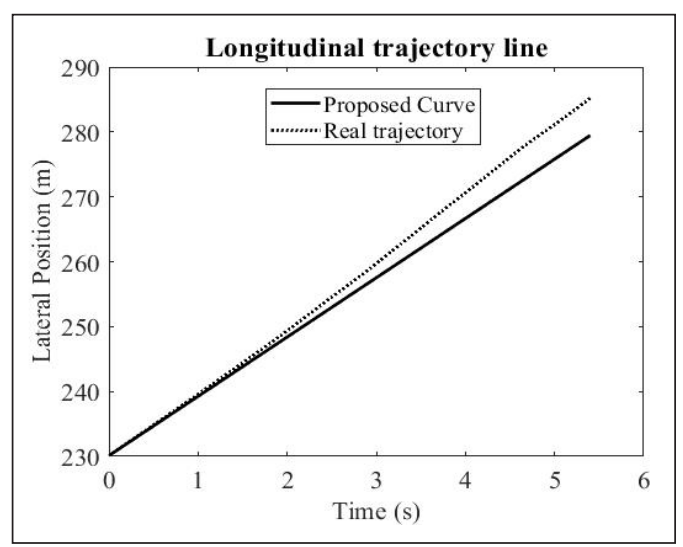

Figure 9. Longitudinal movement of RLC 
Similarly, Figure 10 represents the lateral displacements of our proposed curve, HTC, SC and real trajectory of a vehicle (randomly selected from 100 groups of vehicles) for LLC. Figure 11 shows the Wang et al. (2018) proposed longitudinal movements and real vehicle longitudinal movements. Figure 10 also clearly shows that the lateral displacements of parametric curve are similar to that of the real trajectory curve, and better than the HTC and SC due to similar reason as mentioned above for Figure 8. This research does not consider any modification model for longitudinal movements.

\section{Velocity and Acceleration Profiles Validation}

Recently, Ali et al. (2019), Gu et al. (2019), and Li et al. (2016) applied the data smoothing process for using NGSIM data on other traffic researches. The velocity and acceleration data cannot be applied directly in any model because they have many noises. In addition, these two trajectories were derived from positional information (Thiemann et al., 2008). In this reason, the above-mentioned studies employed the data smoothing technique. Therefore, for validation testing of velocity and acceleration profiles, this study applied the splinebased interpolation by using MATLAB Curve Fitting Package. This similar procedure was applied for trajectory model testing by Wang et al. (2018).

The Equation 4 represents the simulated lateral velocity, and real lateral movements generated the real lateral velocity wherein every LC vehicle has velocity and acceleration trajectories. To test the validation, this study compared the simulated and real lateral velocities and accelerations by using RMSE value. The validation tests considered 100 LLC vehicles and 100 RLC vehicles. These trajectories were formed in a same time frame. Therefore, every LC vehicle adopted the RMSE value whereas Table 2 shows the average RMSE values in which proposed velocity and acceleration models were better than PCbased velocity and acceleration models.

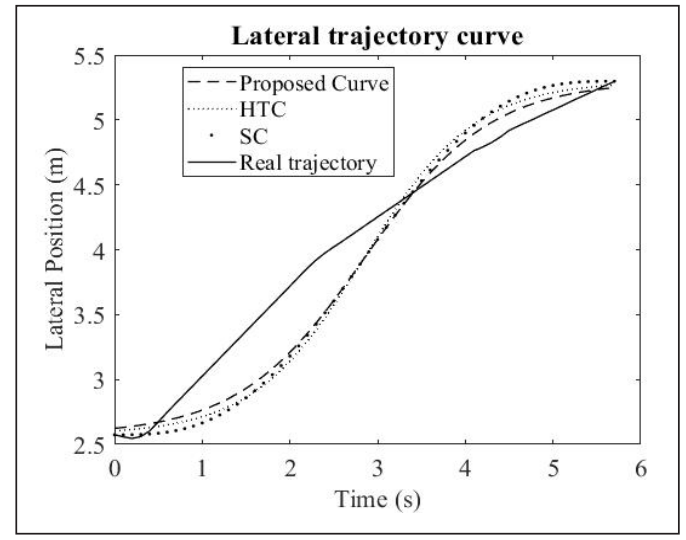

Figure 10. Lateral movement of LLC

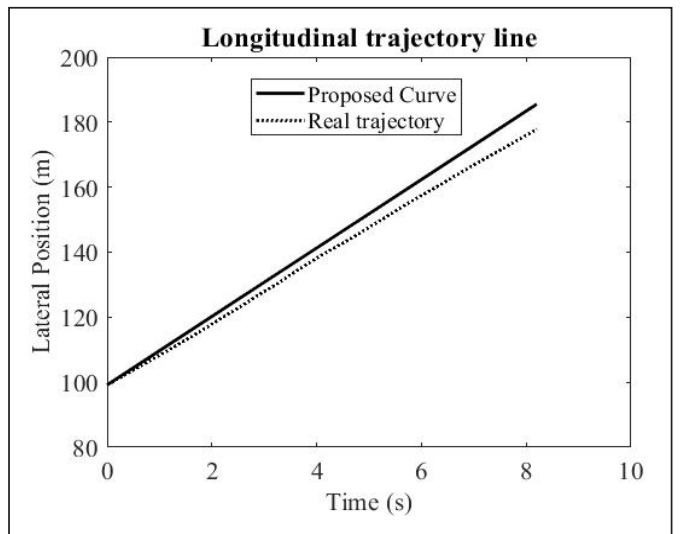

Figure 11. Longitudinal movement of LLC 
The test results for our proposed model revealed that respectively, the average RMSE of velocity and acceleration were 0.218 and 0.314 for LLC, and 0.148 and 0.238 for RLC. Respectively, the average RMSE for PC model of velocity and acceleration were 0.236 and 0.362 for LLC, and 0.188 and 0.302 for RLC. Therefore, the proposed model improved from PC model $7.75 \%$ (velocity profile) and 13.28\% (acceleration profile) for LLC, and $21.43 \%$ (velocity profile) and $21.13 \%$ (acceleration profile) for RLC.

Table 2

Comparative validation test results for velocity and acceleration profiles

\begin{tabular}{lcccc}
\hline Model & \multicolumn{2}{c}{ RMSE(LLC) } & 100 vehicles & \multicolumn{2}{c}{ RMSE (RLC) 100 vehicles } \\
\hline Profile & Velocity & Acceleration & Velocity & Acceleration \\
\hline Proposed & 0.218 & 0.314 & 0.148 & 0.238 \\
PC & 0.236 & 0.362 & 0.188 & 0.302 \\
Improved proposed model (\%) & $7.75 \%$ & $13.28 \%$ & $21.43 \%$ & $21.13 \%$ \\
\hline
\end{tabular}

The second challenge of Katrakazas et al. (2015) adopted in introduction of this study was to test the validation against real data set. Table 2 shows the validation test results using real data and comparisons with PC-based velocity and acceleration models. This section also shows the graphical comparison among real data, proposed simulation model, and PC model for velocity and acceleration profiles. Therefore, Figure 12 (LLC) and Figure 13 (RLC) show the velocity profiles of proposed lateral movements using Equations 4, 11 (Appendix C) and real vehicle data.

Our proposed velocity was smoothly increasing before the middle of the lane and smoothly decreasing after the middle of the lane as shown in Figure 12 (LLC) and Figure 13 (RLC). The dashed line represents proposed model velocity, dotted line represents PC model velocity while solid line represents real velocity (Figures 12 and 13). So, the proposed velocity profile fits more than PC model with real data. In addition, the longitudinal velocity is almost constant velocity because of linear movements, a similar result showed by Chebly et al. (2017). Figures 12 and 13 show that the velocities at starting and ending points were not always zeros for real vehicle, whereas PC model considered zeros at these points. This similar statement was proven by Yang et al. (2018).

Further, Figure 14 (LLC) and Figure 15 (RLC), show the lateral accelerations of proposed lateral movements of Equations 6, 12 (Appendix C) and real vehicle. Although, the longitudinal acceleration of prediction movements was zero shown in Equation 5 due to the constant longitudinal velocity as like Chebly et al. (2017). So, the acceleration of simulated vehicle depended on only lateral acceleration where the lateral acceleration of our proposed model was smooth and flexible. The accelerations at starting and ending points were non-zeros, although PC model considered zeros at these points shown in Figures 14 and 15 . Therefore, the proposed model of velocity and acceleration profiles referred better than PC model as fitted with real trajectory. 


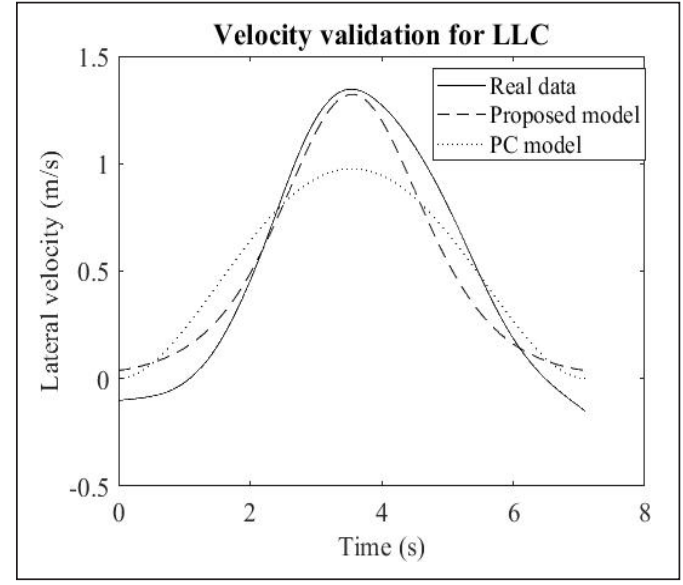

Figure 12 Velocity profiles

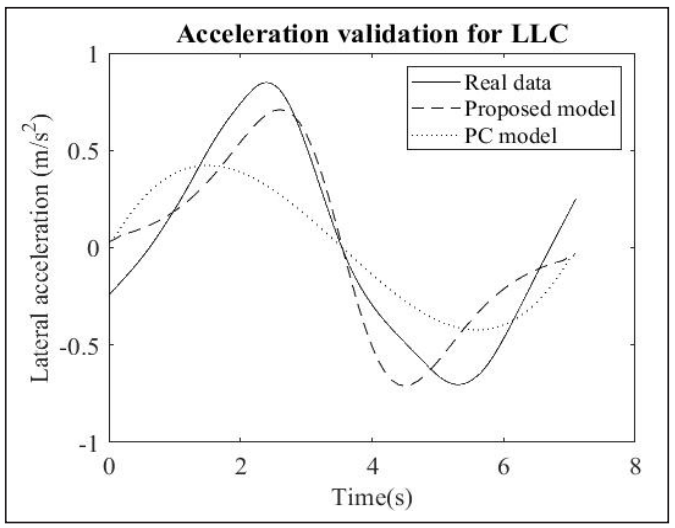

Figure 14. Acceleration profiles

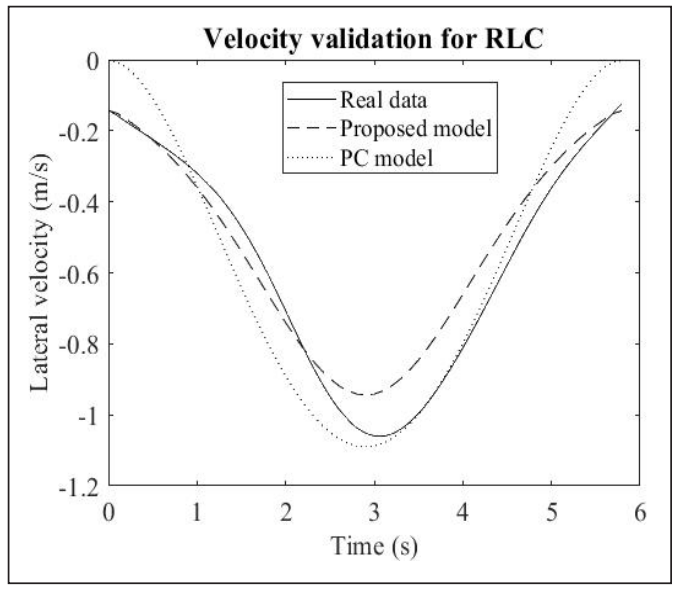

Figure 13 Velocity profiles

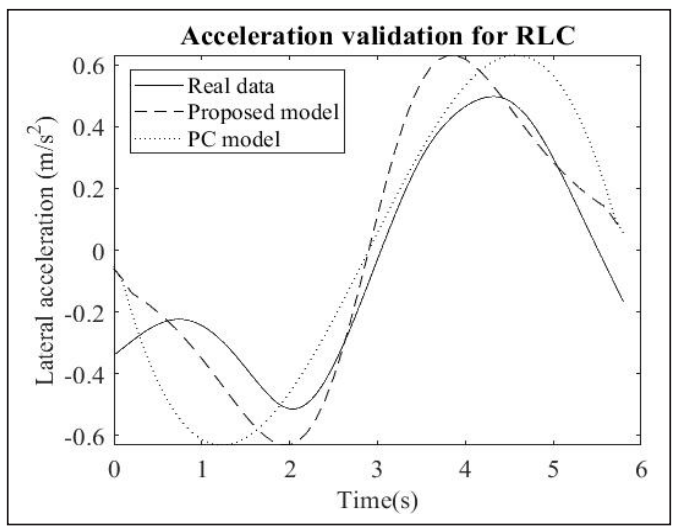

Figure 15. Acceleration profiles

\section{CONCLUSION}

The dynamical systems such as position movement, lateral velocity, lateral acceleration and curvature can be simplified by adopting the LCT planning model. Katrakazas et al. (2015) explored the challenges such as more fitted geometric curve, the continuous and zero-based curvatures at the initial position and ending position, and more realistic velocity and acceleration profiles. The curvatures of the proposed curve for LLC and RLC are lower than the HTC and SC curvatures. In addition, the curvature at LC starting and ending positions are very small and near zeros. Although, some previous LCT models were developed and tested by simulated vehicles, but our model is tested with 200 real vehicles, and shown the significant improvement. The validation test results show that the average RMSE of proposed trajectory model decreases with $1.84 \%$ for LLC and $15.48 \%$ for RLC compared to HTC model and $1.74 \%$ for LLC and $15.60 \%$ for RLC compared to SC model. Furthermore, the proposed model improved from PC model 7.75\% (velocity 
profile) and $13.28 \%$ (acceleration profile) for LLC, and $21.43 \%$ (velocity profile) and $21.13 \%$ (acceleration profile) for RLC. Therefore, the proposed model adopted longitudinal and lateral position trajectory and velocity and acceleration profiles overcome challenges of Katrakazas et al. (2015) work. The lateral trajectory model includes a weighted parameter as aggressive driving behavior tested manually in some fixed values. Therefore, future research should purpose findings optimum parameters by using more real trajectory data.

\section{ACKNOWLEDGEMENT}

All the authors acknowledge Universiti Science Malaysia (School of Mathematical Sciences) and Mawlana Bhashani Science and Technology University for funding this research.

\section{REFERENCES}

Ali, Y., Zheng, Z., Haque, M., \& Wang, M. (2019). A game theory-based approach for modelling mandatory lane- changing behaviour in a connected environment. Transportation Research Part C, 106(February), 220-242. doi: https://doi.org/10.1016/j.trc.2019.07.011

Chebly, A., Talj, R., \& Charara, A. (2017, October 16-19). Maneuver planning for autonomous vehicles, with clothoid tentacles for local trajectory planning. In 20th IEEE International Conference on Intelligent Trans- Portation (ITSC 2017) (pp. 1-6). Yokohama, Japan. doi: 10.1109/ITSC.2017.8317856

Connors, J., \& Elkaim, G. (2007, April 22-25). Analysis of a spline based, obstacle avoiding path planning algorithm. In IEEE Vehicular Technology Conference (pp. 2565-2569). Dublin, Ireland. doi: https://doi. org/10.1109/VETECS.2007.528

Dong, C., Zhang, Y., \& Dolan, J. M. (2017, September 24-28). Lane-change social behavior generator for autonomous driving car by non-parametric regression in reproducing kernel hilbert space. In IEEE International Conference on Intelligent Robots and Systems, 2017-Septe (pp. 4489-4494). Vancouver, BC, Canada. doi: https://doi.org/10.1109/IROS.2017.8206316

González, D., Pérez, J., Milanés, V., \& Nashashibi, F. (2016). A review of motion planning techniques for automated vehicles. IEEE Transactions on Intelligent Transportation Systems, 17(4), 1135-1145. doi: https://doi.org/10.1109/TITS.2015.2498841

Gu, X., Yu, J., Han, Y., Han, M., \& Wei, L. (2019, July 12-14). Vehicle lane change decision model based on random forest. In 2019 IEEE International Conference on Power, Intelligent Computing and Systems, ICPICS 2019 (pp. 115-120). Shenyang, China. doi: https://doi.org/10.1109/ICPICS47731.2019.8942520

Heil, T., Lange, A., \& Cramer, S. (2016, November 1-4). Adaptive and efficient lane change path planning for automated vehicles. In 2016 IEEE 19th International Conference on Intelligent Transportation Systems (ITSC) (pp. 479-484). Rio de Janeiro, Brazil. doi: https://doi.org/10.1109/ITSC.2016.7795598

Katrakazas, C., Quddus, M., Chen, W. H., \& Deka, L. (2015). Real-time motion planning methods for autonomous on-road driving: State-of-the-art and future research directions. Transportation Research Part C: Emerging Technologies, 60, 416-442. doi: https://doi.org/10.1016/j.trc.2015.09.011 
Kawabata, K., Ma, L., Xue, J., \& Zheng, N. (2013, July 9-12). A path generation method for automated vehicles based on Bezier curve. In 2013 IEEE/ASME International Conference on Advanced Intelligent Mechatronics: Mechatronics for Human Wellbeing, AIM 2013 (pp. 991-996). Wollongong, NSW, Australia. doi: https://doi.org/10.1109/AIM.2013.6584223

Léger, J. C. (1999). Menger curvature and rectifiability. Annals of Mathematics, 149(3), 831-869. doi: https:// doi.org/10.2307/121074

Li, L., Chen, X. M., \& Zhang, L. (2016). A global optimization algorithm for trajectory data based car-following model calibration. Transportation Research Part C: Emerging Technologies, 68, 311-332. doi: https:// doi.org/10.1016/j.trc.2016.04.011

Ntousakis, I. A., Nikolos, I. K., \& Papageorgiou, M. (2016). Optimal vehicle trajectory planning in the context of cooperative merging on highways. Transportation Research Part C, 71, 464-488. doi: https://doi. org/10.1016/j.trc.2016.08.007

Resende, P., \& Nashashibi, F. (2010, September 19-22). Real-time dynamic trajectory planning for highly automated driving in highways. In IEEE Conference on Intelligent Transportation Systems, Proceedings, ITSC (pp. 653-658). Funchal, Portugal. doi: https://doi.org/10.1109/ITSC.2010.5625194

Sanchez-Reyes, J., \& Chacón, J. M. (2018). Representation of polynomial nonparametric B e transition curves. Journal of Surveying Engineering, 144(2), 1-8. doi: https://doi.org/10.1061/(ASCE)SU.19435428.0000251

Shen, P., Zhang, X., \& Fang, Y. (2017). Essential properties of numerical integration for time-optimal pathconstrained trajectory planning. IEEE Robotics and Automation Letters, 2(2), 888-895. doi: https://doi. org/10.1109/LRA.2017.2655580

Thiemann, C., Treiber, M., \& Kesting, A. (2008). Estimating acceleration and lane-changing dynamics from next generation simulation trajectory data. Transportation Research Record, 2088(1), 90-101. https:// doi.org/10.3141/2088-10

Wan, Q., Peng, G., Li, Z., Hiroshi, F., \& Inomata, T. (2020). Spatiotemporal trajectory characteristic analysis for traffic state transition prediction near expressway merge bottleneck. Transportation Research Part C, 117, 1-24. doi: https://doi.org/10.1016/j.trc.2020.102682

Wang, C., \& Zheng, C. Q. (2013). Lane change trajectory planning and simulation for intelligent vehicle. In Y. Huang, T. Bao \& H. Wang (Eds.), Advanced materials research (Vol. 671-674, pp. 2843-2846). New York, USA: Trans Tech Publications Ltd. doi: https://doi.org/10.4028/www.scientific.net/AMR.671-674.2843

Wang, Y. Y., Pan, D., Liu, Z., \& Feng, R. (2018). Study on lane change trajectory planning considering of driver characteristics (No. 2018-01-1627). SAE Technical Papers. doi: https://doi.org/10.4271/2018-01-1627

Yang, D., Zheng, S., Wen, C., Jin, P. J., \& Ran, B. (2018). A dynamic lane-changing trajectory planning model for automated vehicles. Transportation Research Part C: Emerging Technologies, 95(June), 228-247. doi: https://doi.org/10.1016/j.trc.2018.06.007

You, F., Zhang, R., Lie, G., Wang, H., Wen, H., \& Xu, J. (2015). Expert systems with applications trajectory planning and tracking control for autonomous lane change maneuver based on the cooperative vehicle infrastructure system. Expert Systems With Applications, 42(14), 5932-5946. doi: https://doi.org/10.1016/j. eswa.2015.03.022 
Zhou, B., Wang, Y., Yu, G., \& Wu, X. (2017). A lane-change trajectory model from drivers' vision view. Transportation Research Part C: Emerging Technologies, 85(October), 609-627. doi: https://doi. org/10.1016/j.trc.2017.10.013 


\section{APPENDIX}

\section{Hyperbolic Tangent curve-based path planning approach:}

The HTC model was proposed by Zhou et al. (2017) converted in Equation 8 using mathematical transformation. The lateral movements of vehicle represented by $Y_{z}(t)$ with respect to time $t$.

$$
\mathrm{Y}_{\mathrm{z}}(\mathrm{t})=\frac{\mathrm{S}_{\mathrm{f}}}{2}\left(\tanh \left(\frac{\sigma}{\mathrm{I}_{\mathrm{d}} / \mathrm{v}_{\mathrm{d}}}\left(\frac{\mathrm{l}_{\mathrm{d}}}{2 \mathrm{v}_{\mathrm{d}}}-\mathrm{t}\right)\right)+\mathrm{T}_{\mathrm{f}}\right)
$$

where, $S_{\mathrm{f}}$ is a scale factor, $\sigma$ is driver aggressiveness, $1_{\mathrm{d}}$ is total longitudinal distance, $\mathrm{V}_{\mathrm{d}}$ average velocity and $\mathrm{T}_{\mathrm{f}}$ is a translation factor during $\mathrm{LC}$ action, where parameters are generated from dynamical trajectories except $\sigma$. This research tested four values $(3,4.3,4.5,7)$ of $\sigma$ according to Zhou et al. (2017) suggestion. The optimized parameter value is 4.5 .

\section{Sine curve-based path planning approach:}

Wang et al. (2018) proposed Equation 9 of lateral movements $\mathrm{Y}_{w}(\mathrm{t})$, where $\mathrm{t}$ is time.

$$
Y_{w}(t)=T_{1} \frac{t}{t_{1}}-\frac{T_{l}}{2 \pi} \sin \left(2 \pi \frac{t}{t_{1}}\right)+T_{f}
$$

where, $\mathrm{T}_{1}$ is total lateral displacement, $\mathrm{t}_{1}$ is total $\mathrm{LC}$ time and $\mathrm{T}_{\mathrm{f}}$ is a translation factor, where all parameters are produced from dynamical trajectories.

\section{Polynomial curve-based path planning approach:}

Chebly et al. (2017) proposed Equation 10 of lateral movements $Y_{p}(t)$, where $t$ is time.

$$
Y_{p}(t)=10 T_{l} \frac{t^{3}}{t 1^{3}}-15 T_{l} \frac{t^{4}}{t 1^{4}}+6 T_{l} \frac{t^{5}}{t 1^{5}}+T_{f}
$$

where, $\mathrm{T}_{1}$ is total lateral displacement, $\mathrm{t}_{1}$ is total $\mathrm{LC}$ time and $\mathrm{T}_{\mathrm{f}}$ is a translation factor, where all parameters are created from dynamical trajectories.

The lateral velocity profile is derived from Equation 10 shown in Equation 11:

$$
V_{p}(t)=30 T_{1} \frac{t^{2}}{t 1^{3}}-60 T_{1} \frac{t^{3}}{t 1^{4}}+30 T_{1} \frac{t^{4}}{t 1^{5}}
$$

The lateral acceleration profile is shown in Equation 12: (Chebly et al., 2017)

$$
A_{p}(t)=60 T_{1} \frac{t}{t 1^{3}}-180 T_{l} \frac{t^{2}}{t 1^{4}}+150 T_{l} \frac{t^{3}}{t 1^{5}}
$$

